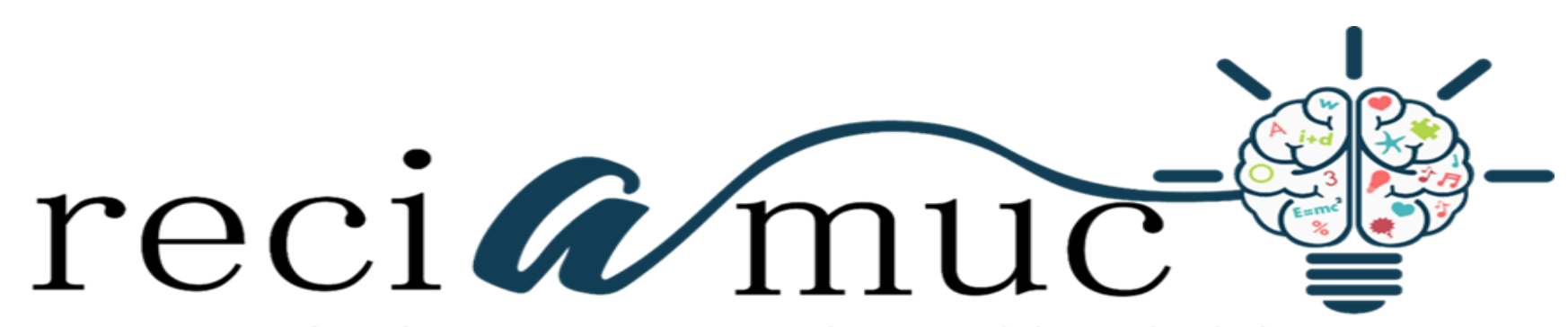

Revista cientifica de investigación actualización del mundo de las ciencias

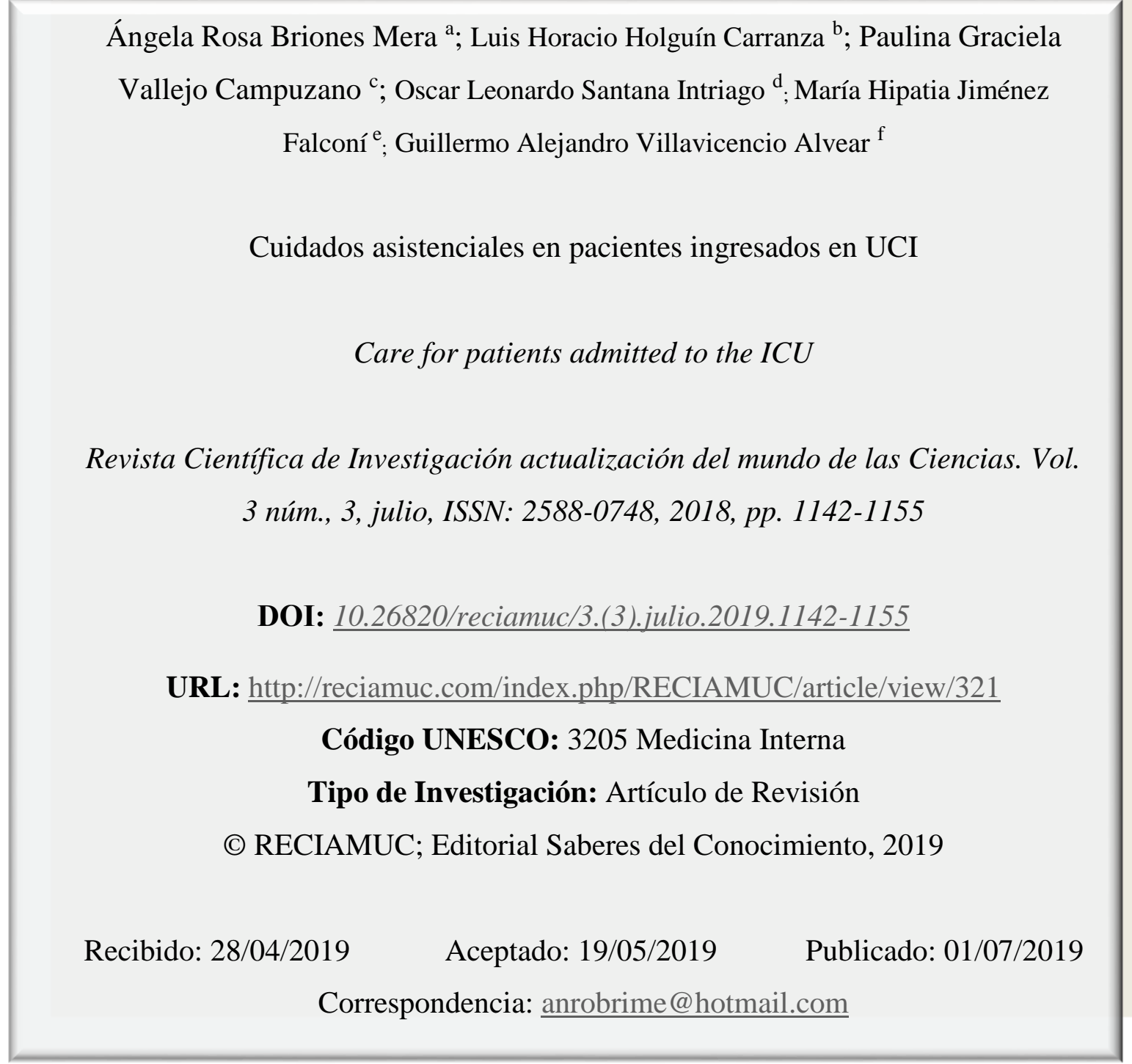

a. Licenciada en Enfermeria; Magister en Investigacion Clinica y Epidemiologia; - Docente de la Facultad de Ciencias de la Salud - Escuela de Enfermería - Universidad Técnica de Manabí; anrobrime@ hotmail.com; Portoviejo, Ecuador.

b. Médico Cirujano; Residente del área de UCI del Hospital General IESS de Manta; 1.holguin@ @otmail.com; Manta, Ecuador.

c. Médico Cirujano; paulina_vallejo@ hotmail.com; Manta, Ecuador.

d. Médico Cirujano; Medico General de primer nivel de atención IESS, Centro de Salud Tipo A; leonardoaries87@ @otmail.com; Calceta, Ecuador.

e. Médico Residente - Hospital IESS Quito Sur - Hospitalización Pediatría; maryjimenez16@ @otmail.com; Quito, Ecuador.

f. Médico General; Médico Residente Asistencial Hospital Carlos Andrade Marín; alex_villavicencio@ hotmail.es; Quito, Ecuador. 


\section{Cuidados asistenciales en pacientes ingresados en UCI}

Vol. 3, núm. 3., (2019)

Ángela Rosa Briones Mera; Luis Horacio Holguín Carranza; Paulina Graciela Vallejo Campuzano; Oscar Leonardo Santana Intriago; María Hipatia Jiménez Falconí; Guillermo

Alejandro Villavicencio Alvear

\section{RESUMEN}

Los cuidados asistenciales en las unidades de cuidados intensivos son fundamentales para mantener la supervivencia de los pacientes en estado crítico y evitar su empeoramiento. Para lograr ese objetivo es esencial que todos los factores involucrados estén en perfecta sintonía, es decir, médicos, personal de enfermería y sistema de salud como tal. Están ampliamente documentados los casos donde factores adversos complican la condición de un paciente crítico, estos van desde neumonías por ventilación mecánica, descuido en la aplicación del tratamiento hasta una simple caída del paciente. La investigación es de tipo revisión bibliográfica que se fundamenta en investigaciones, del mismo tema en medios electrónicos. El trabajo confirma que los malos protocolos de manejos por el personal de enfermería pueden causar el agravamiento de pacientes críticos, en las siguientes áreas 1. Cuidados de enfermería (ulceras, no aplicación de cuidados pautados, flebitis, caída accidental). 2. Mal manejo de vía aérea. 3. Eventos relacionados a manejo acceso vasculares (desconexión catéter central, desconexión sonda nasogástrica) y 4. Infecciones asociadas al cuido (Neumonía asociada a ventilación, Bacteriemia de catéter, infección Urinaria por Sonda Uretral). A su vez es necesario siempre contar con personal altamente calificado y que haya una relación profunda entre el médico y el personal de enfermería en los protocolos de atención a los pacientes críticos en UCI.

Palabras Claves: UCI; Enfermería; Protocolos; Critico; Médicos. 


\section{Cuidados asistenciales en pacientes ingresados en UCI}

Vol. 3, núm. 3., (2019)

Ángela Rosa Briones Mera; Luis Horacio Holguín Carranza; Paulina Graciela Vallejo

Campuzano; Oscar Leonardo Santana Intriago; María Hipatia Jiménez Falconí; Guillermo

Alejandro Villavicencio Alvear

\section{ABSTRACT}

The care in intensive care units is essential to maintain the survival of critically ill patients and prevent their worsening. To achieve this goal it is essential that all the factors involved are in perfect harmony, that is, doctors, nurses and health system as such. Cases where adverse factors complicate the condition of a critical patient are widely documented, ranging from mechanical ventilation pneumonia, carelessness in the application of treatment to a simple fall of the patient. The research is a bibliographic review based on research of the same topic in electronic media. The work confirms that poor management protocols by nurses can cause the aggravation of critical patients in the following areas 1. Nursing care (ulcers, non-application of prescribed care, phlebitis, accidental fall). 2. Bad airway management. 3. Events related to vascular access management (central catheter disconnection, nasogastric tube disconnection) and 4. Infections associated with care (Pneumonia associated with ventilation, catheter bacteraemia, urinary tract infection). At the same time, it is always necessary to have highly qualified personnel and that there is a deep relationship between the doctor and the nursing staff in the protocols of care for critical patients in the ICU.

Key Words: ICU; Nursing; Protocols; Critical; Doctors. 


\section{Cuidados asistenciales en pacientes ingresados en UCI}

Vol. 3, núm. 3., (2019)

Ángela Rosa Briones Mera; Luis Horacio Holguín Carranza; Paulina Graciela Vallejo

Campuzano; Oscar Leonardo Santana Intriago; María Hipatia Jiménez Falconí; Guillermo

Alejandro Villavicencio Alvear

\section{Introducción.}

El paciente crítico es definido por la Sociedad Americana de Medicina Intensiva, como aquel que se encuentra fisiológicamente inestable, que requiere soporte vital avanzado y una evaluación clínica estrecha con ajustes continuos de terapia según evolución. La unidad de cuidados críticos (UCI) es sin duda el lugar que está dotado del personal humano y la infraestructura adecuada para cumplir con los requerimientos de cuidado anteriormente descritos. El análisis de la forma en la que funciona la Red de Atención de Salud, muestra que muchos pacientes críticamente enfermos son evaluados y manejados inicialmente en unidades fuera de la UCI, desde el mundo prehospitalario, servicios de urgencia (SU) y recuperaciones quirúrgicas (Lara, y otros, 2016, págs. 917-918).

La seguridad del paciente, definida por la Alianza Mundial para la Seguridad del Paciente como la reducción del riesgo de daños innecesarios hasta un mínimo aceptable, es un componente constante y en estrecha relación con el cuidado, siendo el profesional de enfermería el protagonista en el proceso de análisis de los riesgos para la consecuente reducción y prevención de incidentes. La preocupación por la seguridad de los pacientes tuvo su marco en la década de 90 con la importante publicación americana ToErr Human: building a safer health system del Institute of Medicine (IOM) donde los autores revelan la muerte de 44.000 a 98.000 americanos al año cuya causa son incidentes en su gran parte prevenibles. Además, el informe pone en discusión la mirada sistémica en el análisis de los eventos versus la cultura del único culpado (Toffoletto \& Ramirez Ruiz, 2013, pág. 1100). Es decir, el empeoramiento de un paciente en cuidados intensivos no solo es provocado por infecciones y desencadenantes de la misma enfermedad, sino que pueden ser causados por el mal manejo del personal que se encarga del monitoreo de los pacientes, que en este caso son el personal de enfermería, sin embargo, puede haber riesgos por las malas decisiones de los médicos tratantes. La Organización Mundial de la Salud (OMS) (2002) establece lineamientos y pautas de actuación para la construcción de políticas encaminadas a prevenir, identificar y minimizar los Eventos Adversos (EA). En congruencia con esta tendencia mundial surge para Latinoamérica el estudio IBEAS (Estudio Ibero Americano de Eventos Adversos) en el que Colombia, Costa Rica, Perú y Argentina se unen para conocer los EA ocurridos en sus 


\section{Cuidados asistenciales en pacientes ingresados en UCI}

Vol. 3, núm. 3., (2019)

Ángela Rosa Briones Mera; Luis Horacio Holguín Carranza; Paulina Graciela Vallejo

Campuzano; Oscar Leonardo Santana Intriago; María Hipatia Jiménez Falconí; Guillermo

Alejandro Villavicencio Alvear

hospitales, establecer su prevalencia y sus características y desarrollar metodologías propias comparables internacionalmente (Achury Saldaña, y otros, 2016, pág. 325).

Los cuidados que deben tener los pacientes ingresados a las unidades de cuidados intensivos (UCI), son muy importantes, para evitar complicaciones que comprometan aún más la vida de los pacientes. En los entornos de clínicas y hospitales en donde existen unidades de cuidados intensivos hay que tener muchas previsiones sanitarias para evitar la proliferación de bacterias e infecciones y a su vez, cuidar el metabolismo de los pacientes para que no haya descompensaciones. En torno a esto (Santana-Cabrera, y otros, 2006) comentan que "El concepto de control de calidad en los cuidados críticos es un tema de cada vez mayor actualidad; evaluar la propia actividad asistencial es necesario en nuestra medicina actual. Uno de estos cuidados es el soporte nutricional; así se han publicado, en los últimos años, trabajos donde se estudian las discrepancias entre el aporte calórico realmente administrado, el pautado por el médico y el teóricamente necesario para el mismo paciente" (pág.662).

Otros autores hablan de la sedoanalgesia como una de las técnicas más importantes del paciente crítico, en este contexto (Estébanez-Montiel, Jiménez-Martín, Sandiumenge, Alonso-Fernández, \& de la SEMICYUC, 2008) hacen mención a que "Una adecuada sedoanalgesia disminuye el grado de estrés del paciente crítico, facilitando su manejo y tratamiento y mejorando su pronóstico. Sin embargo, la sedoanalgesia, sobre todo en perfusión continua, es una práctica que no está exenta de complicaciones derivadas tanto de su infra- como de su sobre-utilización, que pueden empeorar el pronóstico de los pacientes críticos” (pág.19).

Sumado al diagnóstico antes descrito, gran parte de los servicios de urgencia son aún manejados por médicos generales o especialistas con poca formación en urgencia y, por consiguiente, sin mayor preparación para el manejo inicial y menos definitivo de los pacientes más críticos. A pesar de que la especialidad de Medicina de Urgencia ha sido reconocida y existen programas de formación, aún faltan recursos que permitan acortar la brecha de especialistas que puedan hacerse cargo de estos pacientes en el mediano plazo en estos servicios (Lara, y otros, 2016, pág. 918). 


\section{Cuidados asistenciales en pacientes ingresados en UCI}

Vol. 3, núm. 3., (2019)

Ángela Rosa Briones Mera; Luis Horacio Holguín Carranza; Paulina Graciela Vallejo

Campuzano; Oscar Leonardo Santana Intriago; María Hipatia Jiménez Falconí; Guillermo

Alejandro Villavicencio Alvear

Las nuevas tendencias de las tecnologías de información (TICS) están contribuyendo también en los ambientes de urgencias y cuidados intensivos, con la finalidad de tener una mejor organización de los recursos empelados para la atención a los pacientes críticos. Ante esto (Gómez Tello, y otros, 2011) opinan que "El empleo de sistemas de información clínica (SIC) en los SMI persigue mejorar los resultados finales del proceso asistencial, mejorando la seguridad y la calidad, y colaborando en la mejor gestión de los Sistemas de Medicina Interna (SMI). La atención al enfermo crítico consume alrededor del $30 \%$ de los recursos disponibles para el cuidado de enfermos agudos y representa el 8-12\% de los costes monetarios hospitalarios, lo que supone en España más de 2.400 millones de Dólares anuales. La adopción de herramientas que ayuden a gestionar los procesos de cuidados de estos enfermos puede generar una distribución más eficiente de los recursos sanitarios, puesto que los SIC facilitan a tiempo la información para optimizar la toma de decisiones clínicas y de gestión para una asistencia más efectiva y segura (pág. 485-486).

Lo anteriormente descrito también se pudiera llamar como gestión de calidad en la salud, ya incluso la norma internacional ISO 9001:2008 certifica la calidad de la asistencia sanitaria en los centros asistenciales. En este sentido (Lorenzo Torrent, Sánchez Palacios, Santana Cabrera, Cobian Martinez, \& García del Rosario, 2010) hacen referencia a este tema cuando opinan que "El mantenimiento de la calidad, tanto referida a la seguridad como a la eficacia, puede llevarse a cabo mediante un sistema de calidad revisado periódicamente por una certificación externa, según una norma, como la ISO (Internacional Organization for Standardization) 9001, cuya primera versión corresponde al año 1987; actualmente se encuentra en vigor la ISO9001:2008, que hace especial énfasis en el control del cumplimiento de los requisitos legales aplicables al servicio que ofrece la organización certificada. Existen, aproximadamente, un millón de empresas certificadas según ISO 9001 en todo el mundo, y España, con unas 65.000 empresas certificadas, se encuentra en el cuarto lugar, por delante de países como Alemania, Estados Unidos o Gran Bretaña (pág. 477).

\section{Metodología.}

El presente trabajo de investigación está enfocado en una revisión bibliográfica acerca de los procedimientos y cuidados que se deben emplear por los diferentes centros asistenciales y sus 


\section{Cuidados asistenciales en pacientes ingresados en UCI}

Vol. 3, núm. 3., (2019)

Ángela Rosa Briones Mera; Luis Horacio Holguín Carranza; Paulina Graciela Vallejo

Campuzano; Oscar Leonardo Santana Intriago; María Hipatia Jiménez Falconí; Guillermo

Alejandro Villavicencio Alvear

unidades de cuidados intensivos para la óptima atención de los pacientes críticos. Sabiendo que hay un cumulo de factores que interrelacionados entre sí pueden producir complicaciones aún más graves en la condición de pacientes críticos. Para tales efectos se recurrió a medios electrónicos, revistas, entre otros, para la obtención de la información, aquí presentada.

\section{Resultados.}

Tabla 1. Variable primaria. Eventos adversos

\begin{tabular}{|c|c|}
\hline categorías & Definición operativa \\
\hline Medicamentos & $\begin{array}{l}\text { Errores en prescripción, transcripción, } \\
\text { dispensación, preparación, administración y } \\
\text { monitorización. }\end{array}$ \\
\hline Vía aérea y ventilación mecánica & $\begin{array}{l}\text { Obstrucción de la vía área, extubación no } \\
\text { programada, reintubación, desconexión } \\
\text { accidental de la ventilación, progresión del } \\
\text { tubo endotraqueal, atelectasia, barotrauma, } \\
\text { bronco aspiración. }\end{array}$ \\
\hline Accesos vasculares, sondas, tubos, drenajes & $\begin{array}{l}\text { Retiro accidental, o desconexión no } \\
\text { programada. }\end{array}$ \\
\hline El cuidado & $\begin{array}{l}\text { Caída, inmovilización, presencia de úlcera } \\
\text { por presión, no aplicación de cuidados } \\
\text { pautados, otros. }\end{array}$ \\
\hline Infección asociada al cuidado & $\begin{array}{l}\text { Infección por neumonía asociada al } \\
\text { ventilador, bacteremia asociada a catéter, } \\
\text { infección del tracto urinario asociado a } \\
\text { sonda uretral, otros. }\end{array}$ \\
\hline Otros & $\begin{array}{l}\text { Procedimiento, fallo de equipos y pruebas } \\
\text { diagnosticas }\end{array}$ \\
\hline Vía aérea y ventilación mecánica & $\begin{array}{l}\text { Obstrucción de la vía área, extubación no } \\
\text { programada, reintubación, desconexión } \\
\text { accidental de la ventilación, progresión del } \\
\text { tubo endotraqueal, atelectasia, barotrauma, } \\
\text { bronco aspiración. }\end{array}$ \\
\hline Accesos vasculares, sondas, tubos, drenajes & $\begin{array}{l}\text { Retiro accidental, o desconexión no } \\
\text { programada. }\end{array}$ \\
\hline El cuidado & $\begin{array}{l}\text { Caída, inmovilización, presencia de úlcera } \\
\text { por presión, no aplicación de cuidados } \\
\text { pautados, otros. }\end{array}$ \\
\hline Infección asociada al cuidado & $\begin{array}{l}\text { Infección por neumonía asociada al } \\
\text { ventilador, bacteriemia asociada a catéter, }\end{array}$ \\
\hline
\end{tabular}




\section{Cuidados asistenciales en pacientes ingresados en UCI}

Vol. 3, núm. 3., (2019)

Ángela Rosa Briones Mera; Luis Horacio Holguín Carranza; Paulina Graciela Vallejo Campuzano; Oscar Leonardo Santana Intriago; María Hipatia Jiménez Falconí; Guillermo

Alejandro Villavicencio Alvear

\begin{tabular}{|l|l|}
\hline & $\begin{array}{l}\text { infección del tracto urinario asociado a } \\
\text { sonda uretral, otros. }\end{array}$ \\
\hline
\end{tabular}

Fuente: (Achury Saldaña, y otros, 2016, pág. 328).

La presente tabla se puede observar una clasificación de eventos adversos, la cual se obtuvo de un estudio realizado por (Achury Saldaña, y otros, 2016) en 17 unidades de cuidados intensivos (adulto, pediátrico y neonatal) de Colombia, Argentina y México. La muestra de este estudio fueron 1163 eventos adversos ocurridos en dichos países y unidades. En la tabla se pueden observar las categorías de los eventos y sus causales. En la siguiente tabla proveniente del mismo estudio se pueden observar las variables secundarias y una serie de factores relacionados que se pueden presentar.

Tabla 2. Variables secundarias. Factores relacionados

\begin{tabular}{|c|c|}
\hline Variable & Definición operativa \\
\hline \multirow[t]{4}{*}{ Intrínsecos } & Condición clínica. \\
\hline & Factores sociales. \\
\hline & Factores mentales y psicológicos. \\
\hline & Relaciones interpersonales. \\
\hline \multirow[t]{2}{*}{ Extrínsecos } & $\begin{array}{l}\text { Tratamiento y cuidado intrahospitalario } \\
\text { ofrecido por dispositivos y el manejo } \\
\text { terapéutico. }\end{array}$ \\
\hline & $\begin{array}{l}\text { Factores individuales del trabajador: } \\
\text { aspectos físicos y psicológicos. }\end{array}$ \\
\hline \multirow[t]{4}{*}{ Del sistema } & $\begin{array}{l}\text { Factores de equipos y } \text { sociales: } \\
\text { congruencia de rol y liderazgo. }\end{array}$ \\
\hline & $\begin{array}{l}\text { Factores de comunicación: verbal y } \\
\text { escrita. }\end{array}$ \\
\hline & $\begin{array}{l}\text { Factores ligados a la tarea: guías, } \\
\text { protocolos, procedimientos y política, } \\
\text { ayuda a la toma de decisiones, pruebas } \\
\text { complementarias. }\end{array}$ \\
\hline & $\begin{array}{l}\text { Factores ligados a la formación y } \\
\text { entrenamiento: competencia, supervisión, } \\
\text { tiempo. }\end{array}$ \\
\hline
\end{tabular}

Fuente: (Achury Saldaña, y otros, 2016, pág. 329)

Los resultados más importantes obtenidos del estudio antes mencionado refieren que el 34,3\% de los eventos adversos sucedidos fueron causa de cuidados de enfermería (ulceras, no aplicación de 


\section{Cuidados asistenciales en pacientes ingresados en UCI}

Vol. 3, núm. 3., (2019)

Ángela Rosa Briones Mera; Luis Horacio Holguín Carranza; Paulina Graciela Vallejo

Campuzano; Oscar Leonardo Santana Intriago; María Hipatia Jiménez Falconí; Guillermo

Alejandro Villavicencio Alvear

cuidados pautados, flebitis, caída accidental), el 19,1\% eventos relacionados a mal manejo de vía aérea, 16,7\% eventos relacionados a manejo acceso vasculares (desconexión catéter central, desconexión sonda nasogástrica), el 13,1\% eventos relacionados a infecciones asociadas al cuido (Neumonía asociada a ventilación, Bacteriemia de catéter, infección Urinaria por Sonda Uretral), $11 \%$ medicamentos (administración) y un 5,8\% eventos relacionados con otros aspectos como procedimiento y fallos de equipos.

En un estudio realizado por (Toffoletto \& Ramirez Ruiz, 2013) en la unidad de cuidados intensivos de un hospital que es el principal centro de atención de urgencias médicas público de adultos de la región metropolitana de Santiago de chile. Fueron analizados 18 incidentes notificados y relacionados a los cuidados de enfermería ocurridos en el período de enero a marzo de 2012. Los principales resultados de este estudio estuvieron clasificados por dos tipos, los incidentes ocurridos por medicamentos e incidentes causados por retiro de artefactos terapéuticos. A continuación, se presenta la figura 1. Donde se presentan todos los incidentes ocurridos por medicamentos. 
Cuidados asistenciales en pacientes ingresados en UCI

Vol. 3, núm. 3., (2019)

Ángela Rosa Briones Mera; Luis Horacio Holguín Carranza; Paulina Graciela Vallejo Campuzano; Oscar Leonardo Santana Intriago; María Hipatia Jiménez Falconí; Guillermo

Alejandro Villavicencio Alvear

Figura 1. Factores contributivos identificados en el análisis causa raíz de los incidentes relacionados a medicamentos

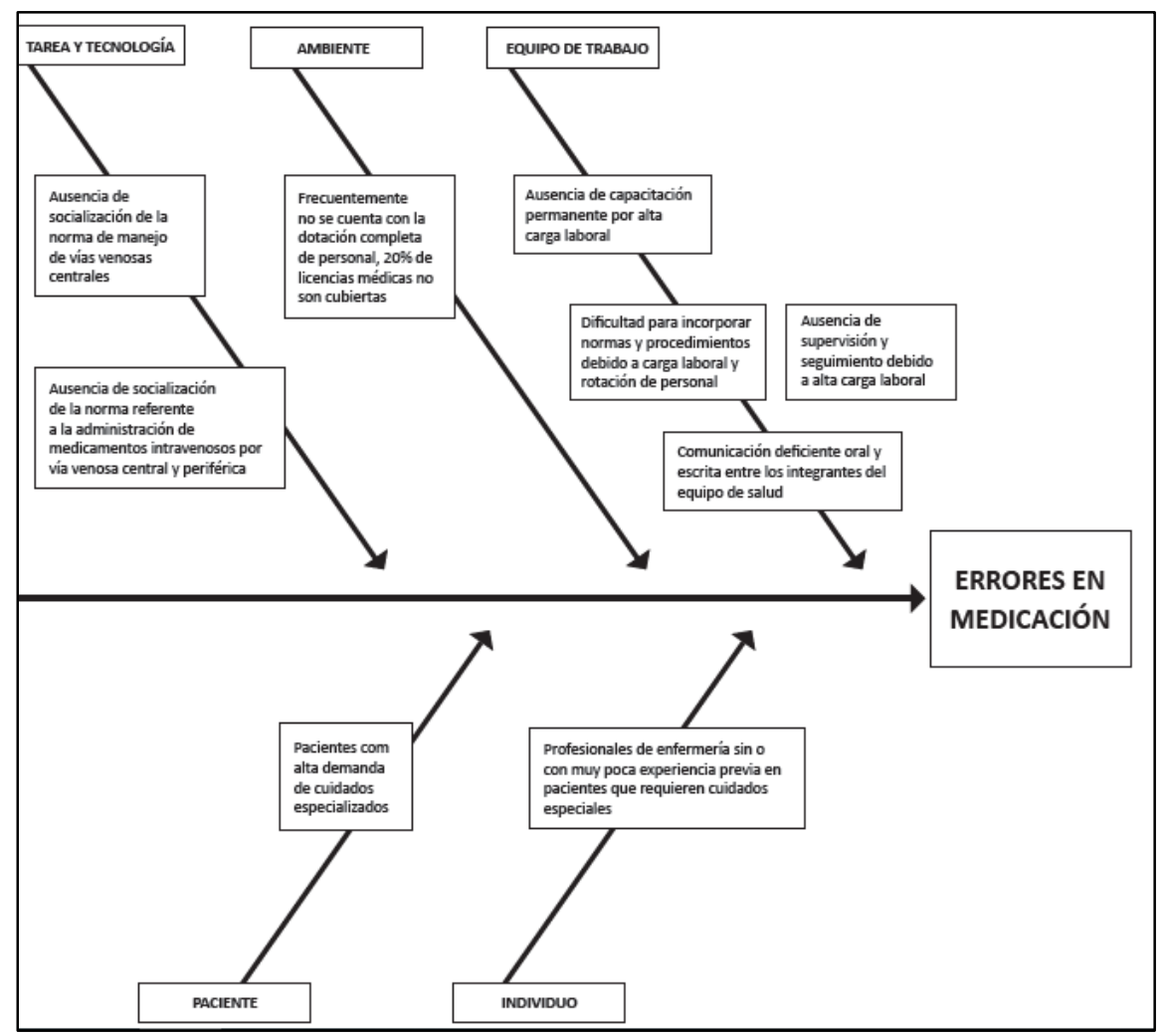

Fuente: (Toffoletto \& Ramirez Ruiz, 2013, pág. 1103)

En la figura 2 se presentan los incidentes relacionados al auto retiro de equipos terapéuticos. 


\section{Cuidados asistenciales en pacientes ingresados en UCI}

Vol. 3, núm. 3., (2019)

Ángela Rosa Briones Mera; Luis Horacio Holguín Carranza; Paulina Graciela Vallejo

Campuzano; Oscar Leonardo Santana Intriago; María Hipatia Jiménez Falconí; Guillermo

Alejandro Villavicencio Alvear

Figura 2. Factores contributivos identificados en el análisis causa raíz de los incidentes relacionados a auto retiro de artefactos terapéuticos

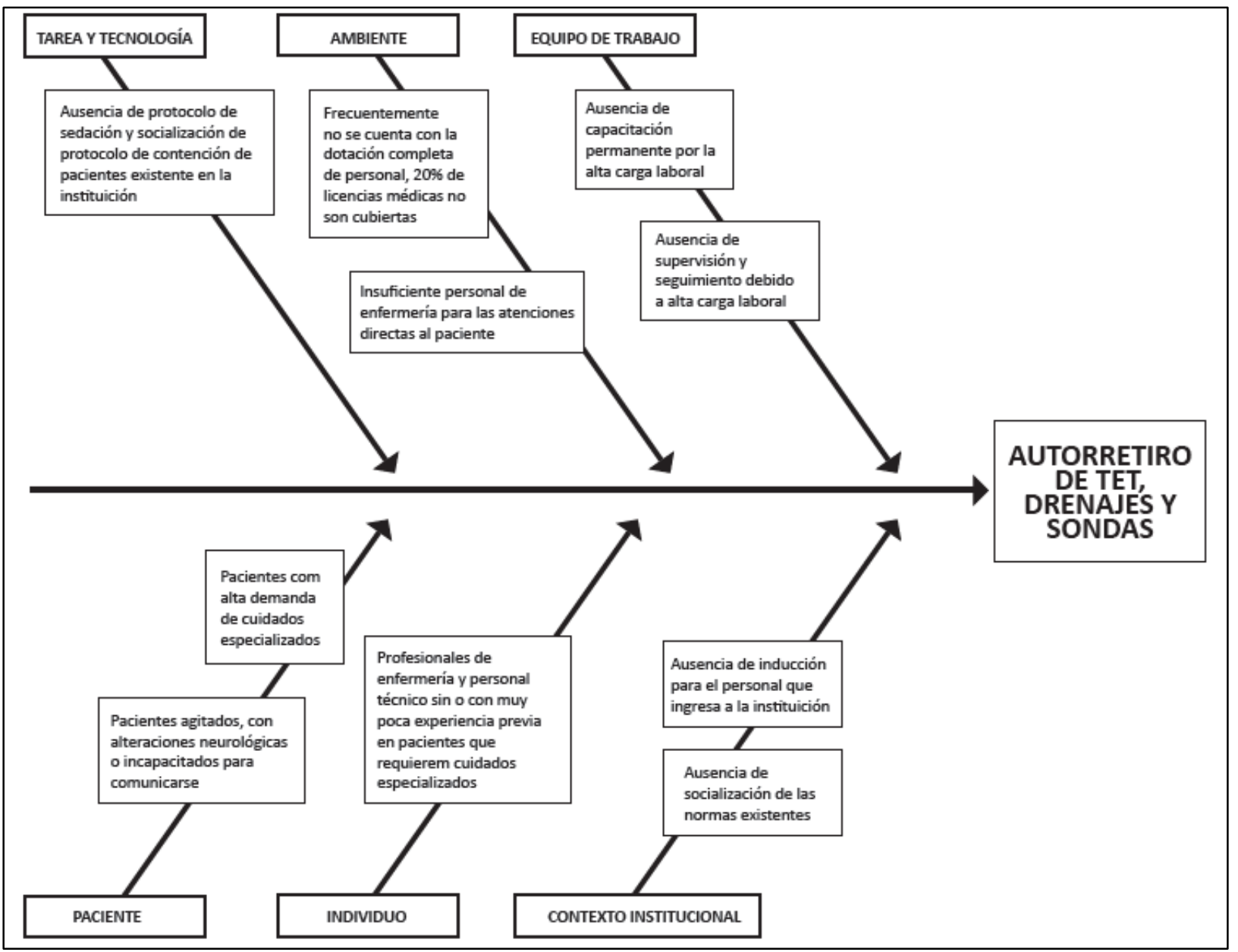

Fuente: (Toffoletto \& Ramirez Ruiz, 2013, pág. 1104).

En el trabajo presentado por (Lara, y otros, 2016) indican que la saturación de los servicios de urgencias en chile, aumentan los errores médicos, un 50\% causan complicaciones graves o muertes, un tercio de ellas ocurren en saturación. El gran causante de los errores es la omisión del tratamiento, los tiempos de atención a los pacientes en urgencias son vitales y en ambientes saturados ese tiempo disminuye complicando aún más la efectividad en la atención médica.

(Pérez Morales, Barletta Castillo, Quintana Hernández, Reyes Rodríguez, \& Otero Espino, 2012) Realizaron un estudio en donde analizan los casos de neumonía presentados por causa de la 


\section{Cuidados asistenciales en pacientes ingresados en UCI}

Vol. 3, núm. 3., (2019)

Ángela Rosa Briones Mera; Luis Horacio Holguín Carranza; Paulina Graciela Vallejo

Campuzano; Oscar Leonardo Santana Intriago; María Hipatia Jiménez Falconí; Guillermo

Alejandro Villavicencio Alvear

ventilación en unidades de cuidados intensivos. El estudio fue realizado en el Laboratorio de Microbiología y salas de cuidados intensivos del Hospital General Universitario Dr. Gustavo Aldereguía Lima, de Cienfuegos, la muestra fue de 104 pacientes ingresados en la UCI. Los resultados más significativos fueron los siguientes. El 9,5\% de los pacientes ventilados sufrió neumonía por ventilación, el $46 \%$ de los pacientes que necesitaron ventilación estaban en la unidad de cuidados intensivos clínicos (UCIC), la mayor cantidad de pacientes que desarrollaron neumonía por ventilación (15\%) estaban en la unidad de cuidados intensivos polivalentes (UCIP). El 50,6 \% de los aislamientos se obtuvo en la Unidad de Cuidados Intensivos Polivalentes. El microorganismo aislado con mayor frecuencia en todas las salas fue Acinetobacter baumannii (41,4\%), con excepción de la Unidad de Cuidados Intensivos Quirúrgicos, unidad en que predominó Pseudomonas aeruginosa, que fue aislada en 11 de los 24 pacientes para un 45,8 \%. Por otra parte, Staphylococcus aureus resultó ser, después de Acinetobacter baumannii, el microorganismo más aislado en la Unidad de Cuidados Intensivos Polivalentes.

\section{Conclusiones.}

Todos los estudios analizados y revisados sobre la atención de pacientes críticos en las unidades de cuidados intensivos, destacan principalmente varios elementos que pueden incidir en empeorar la condición de un paciente crítico. En primer lugar, es muy común, la presencia de neumonía por ventilación, las bacterias más predominantes causales son: Acinetobacter baumannii, Pseudomonas aeruginosa y Staphylococcus aureus. En otros aspectos se identifican los malos protocolos de manejos por el personal de enfermería que han causado el agravamiento de pacientes críticos, en las siguientes áreas 1. Cuidados de enfermería (ulceras, no aplicación de cuidados pautados, flebitis, caída accidental). 2. Mal manejo de vía aérea. 3. Eventos relacionados a manejo acceso vasculares (desconexión catéter central, desconexión sonda nasogástrica) y 4. Infecciones asociadas al cuido (Neumonía asociada a ventilación, Bacteriemia de catéter, infección Urinaria por Sonda Uretral).

Otros aspectos a considerar es que en los países desarrollados hay más cuidado y prevención en las unidades de cuidados intensivos que en Latinoamérica. La sobre saturación de los servicios de 


\section{Cuidados asistenciales en pacientes ingresados en UCI}

Vol. 3, núm. 3., (2019)

Ángela Rosa Briones Mera; Luis Horacio Holguín Carranza; Paulina Graciela Vallejo

Campuzano; Oscar Leonardo Santana Intriago; María Hipatia Jiménez Falconí; Guillermo

Alejandro Villavicencio Alvear

urgencias, lo que ocasiona la proliferación de errores por parte de médicos, el tiempo de espera para atención de pacientes críticos empeora su agravamiento, lo que por supuesto genera una baja calidad en la atención. Otros de los aspectos a considerar es que es necesaria la implementación de sistemas de gestión de calidad en las unidades de cuidado intensivos, esto permite llevar un mejor registro de la enfermedad del paciente, el tratamiento indicado por el médico tratante, el personal de enfermería encargado de aplicar el tratamiento y su rotación correspondiente, reduciendo también los costos operativos de los hospitales o clínicas.

Hay nuevas tecnologías que han estado apareciendo y que ya están siendo aplicadas en muchos países en el mundo. La tele-UCI que es básicamente un sistema que por mando a distancia y en tiempo real por medio de audio y video se puede monitorear al paciente, dando resultados como: notificación electrónica de los signos vitales, el estado clínico, exámenes, el tratamiento y los equipos utilizados, además de ponerse en contacto con el equipo sanitario de esa UCI, entre otras que ayudan a ofrecer un servicio óptimo diferenciado y de calidad.

Es fundamental que se forme un equipo médico - enfermero(a), en donde quede bien claro los pasos a tomar con cada paciente en particular, y que quede bien claro el tratamiento a aplicar, esto con el fin de evitar confusiones y errores que provoquen situaciones no deseadas para el paciente. La conclusión muy general es que existen muchos factores intrínsecos y extrínsecos que pueden de un instante a otro cambiar la condición médica de un paciente crítico hospitalizado en una unidad de cuidado intensivo.

\section{Bibliografía.}

Achury Saldaña, D., Rodríguez, S., Díaz, J., Cavallo, E., Zarate Grajales, R., Vargas Tolosa, R., $\&$ de las Salas, R. (2016). Estudio de eventos adversos, factores y periodicidad en pacientes hospitalizados en unidades de cuidado intensivo. Enfermería global, 15(42), 324-340.

Estébanez-Montiel, M., Jiménez-Martín, M., Sandiumenge, A., Alonso-Fernández, M., \& de la SEMICYUC, G. (2008). Sedación prolongada en unidades de cuidados intensivos. Medicina Intensiva, 32(Supl. 1), 19-30. 


\section{Cuidados asistenciales en pacientes ingresados en UCI}

Vol. 3, núm. 3., (2019)

Ángela Rosa Briones Mera; Luis Horacio Holguín Carranza; Paulina Graciela Vallejo Campuzano; Oscar Leonardo Santana Intriago; María Hipatia Jiménez Falconí; Guillermo

Alejandro Villavicencio Alvear

Gómez Tello, V., Álvarez Rodríguez, J., Núñez Reiz, A., González Sánchez, J., Hernández Abadía de Barbará, A., Martínez Fresneda, M., \& Calvete Chicharro, M. (2011). Estándares técnicos y funcionales, y proceso de implantación, de un sistema de información clínica en unidades de cuidados intensivos. Medicina intensiva, 35(8), 484-496.

Kuerten Rocha, P., Prado, M., Almeida Cabral, P., de Souza, J., Izabel, A., \& Anders, J. (2013). El cuidado y la tecnología en las unidades de cuidados intensivos. Index de Enfermería, 22(3), 156-160.

Lara, B. A., Cataldo, A., Castro, R., Aguilera, P., Ruiz, C., \& Andresen, M. (2016). Medicina de urgencia y unidades de cuidados intensivos: Una alianza necesaria en busca de la mejoría de la atención de pacientes críticos. Revista médica de Chile, 144(7), 911-917.

Lorenzo Torrent, R., Sánchez Palacios, M., Santana Cabrera, L., Cobian Martinez, J., \& García del Rosario, C. (2010). Gestión de la calidad en una unidad de cuidados intensivos: implementación de la norma ISO 9001: 2008. Medicina intensiva, 34(7), 474-482.

Pérez Morales, L., Barletta Castillo, J., Quintana Hernández, H., Reyes Rodríguez, I., \& Otero Espino, N. (2012). Estudio clínico, epidemiológico y microbiológico de pacientes con neumonía asociada a la ventilación mecánica ingresados en salas de cuidados intensivos. Medisur, 10(4), 268-278.

Santana-Cabrera, L., O'Shanahan-Navarro, G., García-Martul, M., Ramírez Rodríguez, A., Sánchez-Palacios, M., \& Hernández-Medina, E. (2006). Calidad del soporte nutricional artificial en una unidad de cuidados intensivos. Nutrición hospitalaria, 21(6), 661-666.

Toffoletto, M. C., \& Ramirez Ruiz, X. (2013). Mejorando la seguridad de los pacientes: estudio de los incidentes en los cuidados de enfermería.

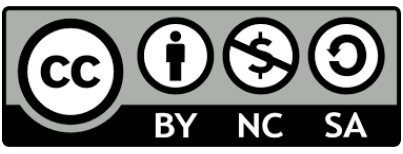

RECONOCIMIENTO-NOCOMERCIAL-COMPARTIRIGUAL

CC BY-NC-SA

ESTA LICENCIA PERMITE A OTROS ENTREMEZCLAR, AJUSTAR Y CONSTRUIR A PARTIR DE SU OBRA CON FINES NO

COMERCIALES, SIEMPRE Y CUANDO LE RECONOZCAN LA AUTORÍA Y SUS NUEVAS CREACIONES ESTÉN BAJO UNA LICENCIA CON LOS MISMOS TÉRMINOS. 